result. The effectiveness of treatment was less in patients $>65$ years (OR $0.418 ; 95 \% \mathrm{Cl} 0.342-0.509, \mathrm{p}<0.001)$, OA stage $\geq 2$ by Kelgren-Lawrence (OR 0.556; 95\% Cl 0.298-0.738, $\mathrm{p}<0.001$ ), with rest pain (OR $0.690 ; 95 \% \mathrm{Cl} 0.596-0.800, \mathrm{p}<0.001$ ), synovitis (OR $0.780 ; 95 \% \mathrm{Cl}$ $0.673-0.900, p=0.001$ ), and sensory symptoms such as burning, pain cold, electric shocks (OR $0.530 ; 95 \% \mathrm{Cl} 0.458-0.613, \mathrm{p}<0.001$ ).

Conclusion: the ASU and KLS combination allows to achieve successful pain control in OA. A number of factors: age $>65$ years, OA stage $\geq 2$ by Kelgren-Lawrence, rest pain, synovitis, and sensory symptoms are associated with the worst result of treatment.

Disclosure of Interests: Elena Pogozheva: None declared, Andrey Karateev: None declared, Alexander Lila Speakers bureau: Pfizer, Inc., MSD Novartis, AbbVie Inc., Celgen Corporation, Biocad, Janssen, UCB, Inc., Vera Amirdzhanova: None declared, Ekaterina Filatova: None declared DOI: 10.1136/annrheumdis-2019-eular.3933

\section{AB0806 EFFICACY MANAGEMENT AND ADHERENCE EVALUATION OF DICLOFENAC IN THE TREATMENT OF KNEE OSTEOARTHRITIS PAIN}

Yudian Qiu, Jianhao Lin, Hu LI, Yunfei Hou. Inst. of Arthritis, Peking Univ. People's Hosp., Beijing, China

Background: The incidence of knee osteoarthritis is increasing day by day. The accompanying pain has seriously affected people's quality of life. No relevant researches have studied the relationship between pain relief and adherence after taking NSAIDS in outpatients.

Objectives: To investigate the effect of diclofenac on pain control of knee osteoarthritis and the relationship between pain relief and medicine adherence. To evaluate the pain relief rate of patients with different initial pain.

Methods: 120 patients with knee osteoarthritis were recruited from the outpatient department of osteoarthritis, Peking University People's Hospital. The population was randomly divided into the experimental and control group. The baseline and follow-up contents were interviews including socio-demographic factors, evaluation of knee pain, WOMAC and MMAS8 questionnaire. The experimental group was given regular follow-up and medication advice, while the control group was only given observational records at the middle and late stages. SPSS25.0 nonparametric $T$ test and one way ANOVA were used to evaluate the efficacy of diclofenac in relieving knee osteoarthritis pain and the relationship between pain relief and adherence.

Results: A total of 120 patients with knee osteoarthritis at baseline were randomly enrolled and 108 patients were followed up. 55 patients in the experimental group and 53 patients in the control group (1) The 2-week adherence of test group/control group was $86.38 \% / 64.78 \%(P<0.01)$, the 6 week adherence of test group/control group was $75.24 \% / 35.22 \%(P<0.01)$. The 2-week and 6-week adherence of test group and control group were significantly different $(P=0.015$ and $P<0.01)$. (2) The pain relief rate at 2 and 6 weeks in the experimental group was $67.56 \%$ and $69.41 \%$, respectively, the pain relief rate was significantly higher than baseline $(\mathrm{P}<0.01)$, but there was no significant difference between the two groups $(P=0.739)$. The pain relief rates at 2 and 6 weeks in the control group were $46.51 \%$ and $29.03 \%$ and was significantly higher than baseline $(P<0.01)$, there was a significant difference between the two groups $(P<$ 0.01). In the evaluation of 2 and 6 weeks, the pain relief rates in the experimental group were significantly different from those in the control group $(\mathrm{P}<0.01)$. (3) The initial pain scores of different degrees did not affect the adherence and pain relief rates in the experimental group. In the control group, the 2-week adherence of severe pain patients was higher than that of mild and moderate pain patients $(P<0.01)$, but the 6 week follow-up adherence shows no significant difference in patients with different degrees of initial pain $(P=0.073)$, the 6-week adherence of patients with moderate/severe pain $(38.07 \% / 42.6 \%)$ was significantly different from the 2-week adherence $(68.34 \% / 84.77 \%) \quad(P<0.01)$, the 6-week pain relief rate $(18.46 \% / 29.72 \%)$ in patients with mild/moderate pain was lower than the 2 -week pain relief rate $(48.72 \% / 47.44 \%)(P=0.052 / P<0.01)$. (4) The patients with better adherence have the higher pain relief rate ( $P$ $<0.01$ ).

Conclusion: For patients suffering from knee osteoarthritis pain, diclofenac $150 \mathrm{mg} /$ day for 6-week is recommended. Regular follow-up can improve adherence significantly, increased adherence can improve the pain relief rate significantly, the pain relief rate may not be related to the patient's initial pain score. It is suggested that clinical workers should supervise the medication of patients with knee osteoarthritis and make regularly follow up.

\section{REFERENCES}

[1] da Costa BR, Reichenbach S, Keller N, et al. Effectiveness of non-steroidal anti-inflammatory drugs for the treatment of pain in knee and hip osteoarthritis: a network meta-analysis. Lancet 2016;387:2093-105

Acknowledgement: Thanks for the support from the outpatient department of Peking University People's Hospital.

Disclosure of Interests: None declared

DOI: 10.1136/annrheumdis-2019-eular.7289

\section{AB0807 1 FOLLOW-UP OF PATIENTSWITH HIP OSTEOARTHRITIS WHO RECEIVED VISCOSUPPLEMENTATION: HOW NEW TECHNOLOGIES CAN HELP}

Mariaeva Romano, Laura Belloli, Elisa Verduci, Cinzia Casu, Valeria Campanella, Marina Muscarà, Davide Antonio Filippini, Emanuela Schito, Maria DI Cicco, Oscar Massimiliano Epis, Samsung Electronic Italia: M.Cammalleri, G.Locatelli MDApp srl, Y.Cardillo, A.Colombini. Grande Ospedale Metropolitano Niguarda, Reumatologia, Milano, Italy

Background: Digital health innovations have opened up several opportunities to help patients with rheumatologic conditions and their treating clinicians in improving routine healthcare.

Objectives: To evaluate the applicability of a wearable smartwatch for monitoring patients with hip osteoarthritis who received viscosupplementation. Samsung Electronics Italia and MDApp srl supported the present study.

Methods: Consecutive patients with hip osteoarthritis who received viscosupplementation with jaluronic acid between November 2017 and May 2018 were enrolled in this observational study. All patients gave their informed consent. A Samsung Gear smartwatch was given to each patient and was worn during the day for measuring daily steps, walking distance, and mean velocity. Patients were asked to wear the smartwatch starting from one week before infiltration (T0), until two weeks before (T1). Patients were assessed with Lequesne index and WOMAC score at T0 and T1.

Results: Overall, 24 patients $(7$ male and 17 female, mean age 44 years-old) were enrolled. Of these, $70 \%$ has worn the smartwatch for most of the time (range $90-100 \%$ ). An improvement of velocity $(45 \%)$, an increase of daily steps $(29 \%)$, and walking distance $(25 \%)$ were seen Similarly, Lequesne index and WOMAC score were consistent with subjective improvement. Most of the patients $(95 \%)$ declared to be satisfied with this digital health study.

Conclusion: Samsung smartwatches proved to be an effective tool for tracking and recording medical parameters linked with perceived general health. Health technologies are more than an accessories and could be useful in chronic musculo-skeletal diseases because they allow to ease remote diagnosis and to monitor patients' symptoms and clinical response. Telemedicine could fill the gap between patients and healthcare, with a reproducible and objective tool, enhancing patients' engagement with the treatment plan. The high patients' satisfaction further support the use of digital health technology.

\section{REFERENCE}

[1] Kataria S, Digital Health: a new dimension in rheumatology patient care. Rheumatol Int. 2018

Disclosure of Interests: Mariaeva Romano Speakers bureau: janssen, Laura Belloli: None declared, Elisa Verduci: None declared, Cinzia Casu: None declared, Valeria Campanella: None declared, Marina Muscarà None declared, Davide Antonio Filippini: None declared, Emanuela Schito: None declared, Maria Di Cicco: None declared, Oscar Massimiliano Epis Speakers bureau: BMS

DOI: 10.1136/annrheumdis-2019-eular.2992 